\title{
Long-segment restrictive tear of radial Artery under ultrasound guide for arteriovenous fistula by PTA:A case report
}

Jing Xie, ${ }^{1}$, Jiangtao Zhu, ${ }^{2}$, Nan Mao, ${ }^{3}$, Sichong Ren, ${ }^{4}$, and junming fan ${ }^{4}$

${ }^{1}$ Chengdu University of Traditional Chinese Medicine

${ }^{2}$ Changzhi Hospital of Traditional Chinese Medicine

${ }^{3}$ Chengdu Medical College The First Affiliated Hospital

${ }^{4}$ Chengdu Medical College

November 7, 2021

\begin{abstract}
Considerable calcification and stenosis frequently occur in the radial artery (RA) in diabetic nephropathy. PTA was performed successfully using balloon to expand and restrictively tear the longitudinal axis of the RA. Serendipity,the method indicates it may be a useful measure of promoting maturation of AVF in diabetic nephropathy
\end{abstract}

\section{Hosted file}

edt-EEN-210923-M8_my\selectlanguage\{ngerman\} manuscript9.30_12013.doc available https://authorea.com/users/444914/articles/544549-long-segment-restrictive-tear-ofradial-artery-under-ultrasound-guide-for-arteriovenous-fistula-by-pta-a-case-report 

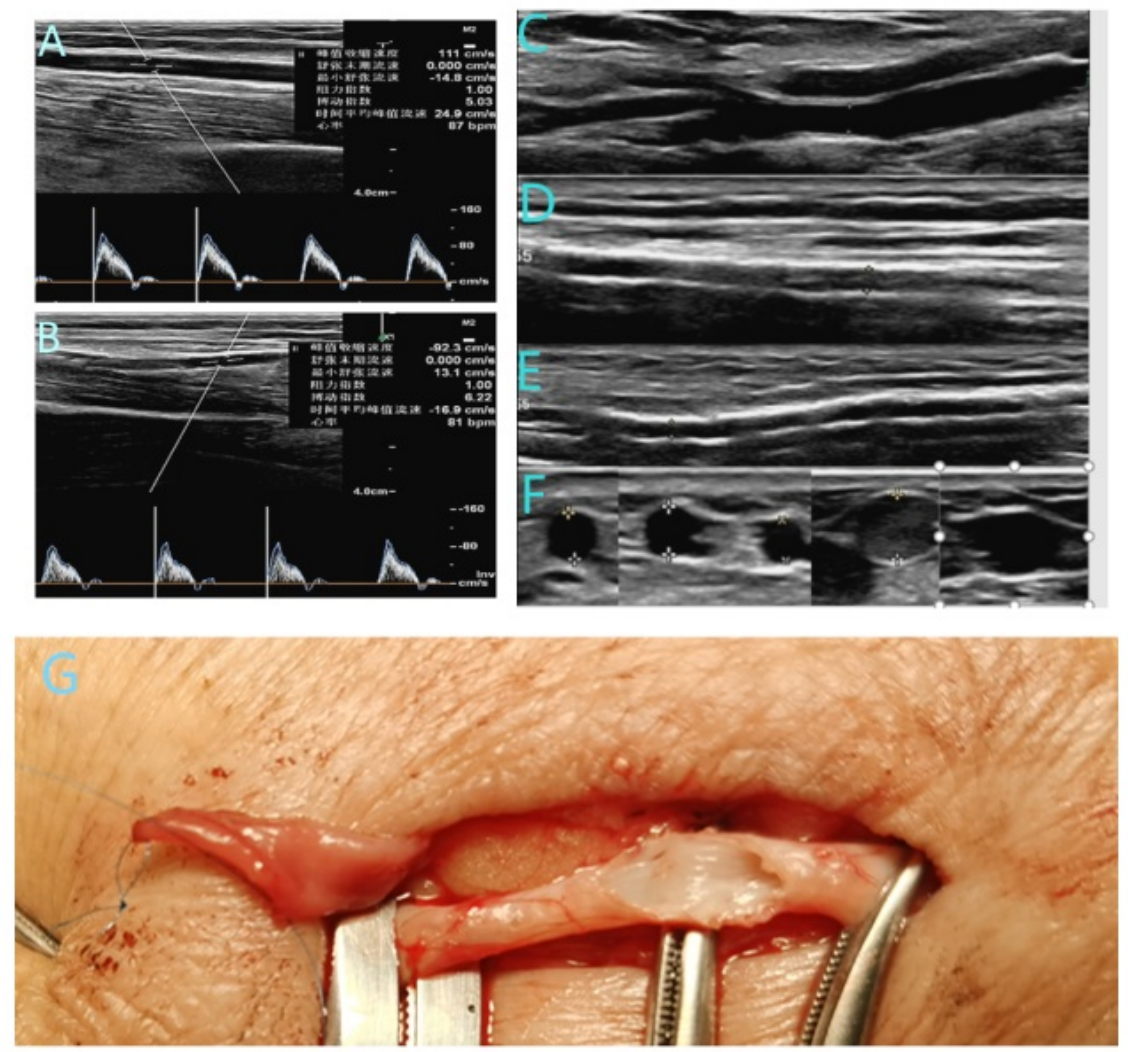


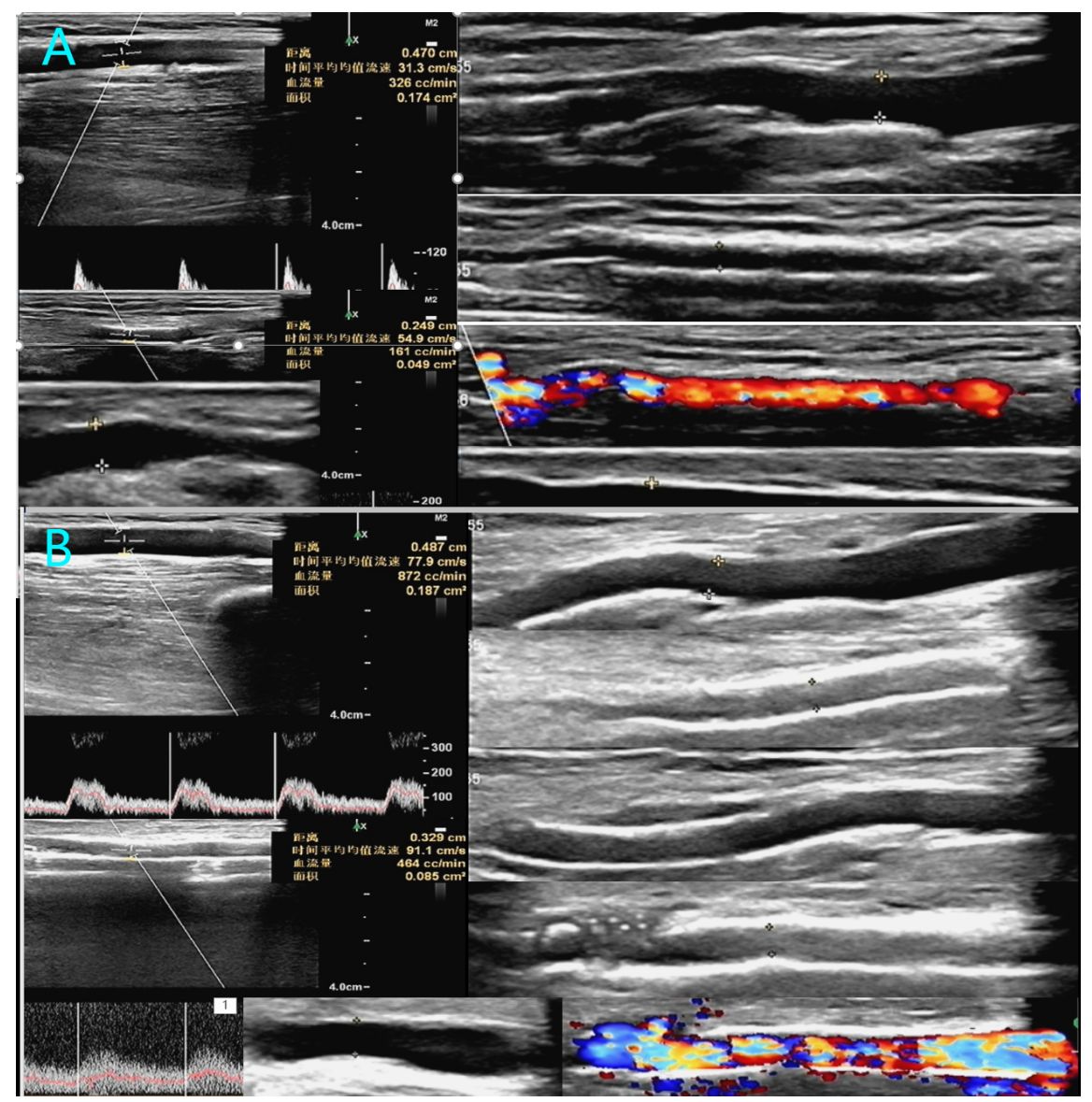



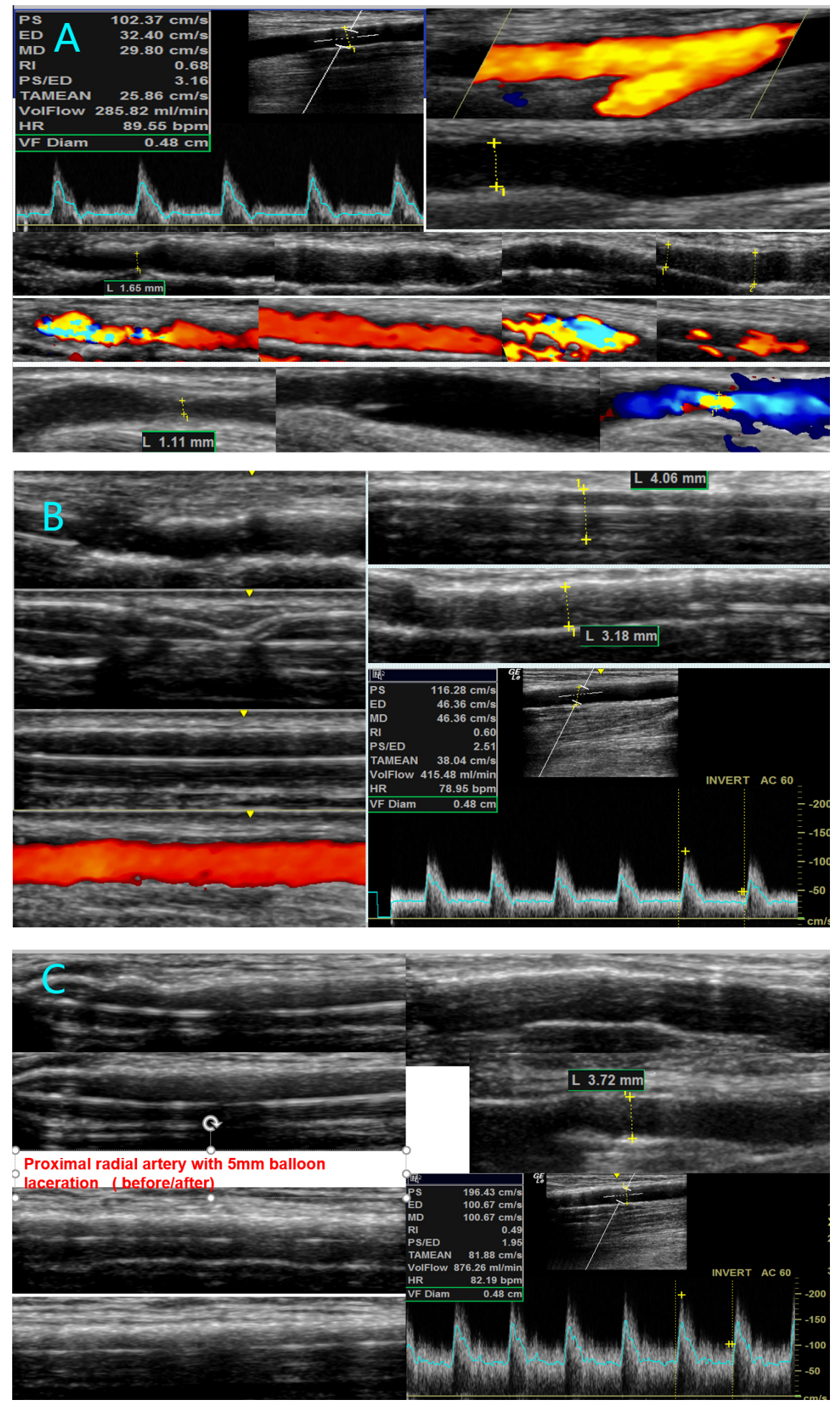